\title{
Urine Clarity Inaccurate to Rule Out Urinary Tract Infection in Women
}

\author{
Amanda Foley, MS, and Linda French, MD
}

Background: A previous study suggested that, among children, a clear urine sample accurately rules out urinary tract infection. The aim of this study was to replicate this previous study done in an adult female population.

Methods: We analyzed 100 anonymous urine samples from female patients ages 18 to 50 years who were receiving care in a university hospital system. Urine samples were tested for clarity by determining if newsprint could be legibly read through them.

Results: Sensitivity, specificity, and positive and negative predictive values for the newsprint test were $13.3 \%, 96.5 \%, 40.0 \%$, and $86.3 \%$, respectively.

Conclusion: Visual inspection of urine clarity was not sufficiently accurate to rule out urinary tract infection in women. (J Am Board Fam Med 2011;24:474-475.)

Keywords: Case Report, Diagnostic Test, Point of Care, Urine Clarity, Urinary Tract Infection

Acute, uncomplicated cystitis represents the most common form of urinary tract infection (UTI) among women. Dipstick urinalysis at the point of care (POC) has been used to improve diagnostic accuracy, but it lacks sensitivity to accurately identify "low count" UTIs. ${ }^{1}$ Thus, additional simple POC testing is desirable.

A previous study among children suggested that urine clarity was an accurate POC test to exclude the diagnosis of UTI. ${ }^{2}$ The authors utilized 159 urine samples from male and female patients ranging in age from 4 weeks to 19 years and found a negative predictive value of more than $97 \%$. The aim of this investigation was to replicate this prior study but in an adult female population.

\section{Materials and Methods}

Between February and May 2009, 100 anonymous urine samples, obtained from adult women, ages 18

This article was externally peer reviewed.

Submitted 12 January 2010; revised 6 January 2011; accepted 10 January 2011.

From the University of Toledo, $\mathrm{OH}$.

Funding: Support for this research has been provided by the University of Toledo.

Conflict of interest: none declared.

Corresponding author: Linda French, University of Toledo, 3000 Arlington Avenue, MS1179, Toledo, OH 43614, E-mail: linda.french@utoledo.edu. to 50 years, in both inpatient and ambulatory locations, were obtained from a medical center laboratory. Institutional review exempted this study and no patient consent was required.

The researcher (AF) obtained samples provided in numbered sterile glass tubes without other patient information. Specimens either were considered fresh by laboratory personnel or were warmed to room temperature after refrigeration before analysis. A page of newsprint was held up against the glass to determine if the print was legible through the sample. Data were recorded as negative if the investigator was able to read the words during the visual test and were recorded as positive if the print was not legible during the visual test.

Each sample was cultured by streaking onto a $5 \%$ sheep's blood agar plate using a $0.001-\mathrm{mL}$ calibrated inoculating loop. The plate was then inverted and placed in an aerobic incubator at 36.1 to $37.9^{\circ} \mathrm{C}$ for 48 hours. Laboratory technicians determined which plates were positive for bacterial growth. Plates were labeled as "no growth" when colonies numbered $<10,000$ colony forming units (CFUs) per milliliter. Growth on a plate that was not caused by UTI-causing bacteria was classified as "no significant growth." A urine culture was classified as "positive growth" when $>100,000$ CFUs of a predominating UTI-causing organism were identified. 
Culture was classified as "low-count positive" when there were $>10,000$ and $<100,000$ CFUs.

Excel 2008 for Macintosh (Microsoft, Redmond, WA) was used to construct a contingency table of the results (Table 1). Sensitivity, specificity, and positive and negative predictive values were calculated.

\section{Results}

Data are displayed in the contingency tables (Tables 1 and 2). Only 5 samples out of the total 100 were positive (illegible) by visual clarity testing. There was only one low-count positive culture (14,000 CFUs) with a corresponding negative clarity test, and 15 additional positive cultures, only 2 of which had a positive clarity test. No significant growth was present in 46 cases, which included the remaining 3 positive clarity tests.

Using a standard cutoff of 100,000 CFUs as positive culture, the sensitivity, specificity, and positive and negative predictive values were calculated as $13.3 \%, 96.5 \%, 86.3 \%$, and $40.0 \%$, respectively. Test performance was even worse using a 10,000CFU cutoff.

\section{Discussion}

The goal of this study was to determine if urine clarity could rule out UTI in women. Because of

Table 1. Contingency table with reference test urine culture $>100,000 \mathrm{CFU}$

\begin{tabular}{cccccc}
\hline & $\begin{array}{c}\text { Positive } \\
\text { Culture }\end{array}$ & $\begin{array}{c}\text { Negative } \\
\text { Culture }\end{array}$ & Totals & $\begin{array}{c}\text { Positive } \\
\text { Predictive } \\
\text { Value }\end{array}$ & $\begin{array}{c}\text { Negative } \\
\text { Predictive } \\
\text { Value }\end{array}$ \\
\hline $\begin{array}{c}\text { Visual test } \\
\text { Positive }\end{array}$ & 2 & 3 & 5 & $2 / 5$ & \\
Negative & 13 & 82 & 95 & & $(40.0 \%)$ \\
Totals & 15 & 85 & 100 & & $(86.3 \%)$ \\
Sensitivity & $2 / 15$ & & & & \\
Specificity & $(13.3 \%)$ & & & & \\
& & $82 / 85$ & & & \\
\hline
\end{tabular}

Table 2. Contingency table with reference test urine culture $>10,000 \mathrm{CFU}$

\begin{tabular}{cccccc}
\hline & $\begin{array}{c}\text { Positive } \\
\text { Culture }\end{array}$ & $\begin{array}{c}\text { Negative } \\
\text { Culture }\end{array}$ & $\begin{array}{c}\text { Positive } \\
\text { Predictive } \\
\text { Talue }\end{array}$ & $\begin{array}{c}\text { Negative } \\
\text { Predictive } \\
\text { Value }\end{array}$ \\
\hline $\begin{array}{c}\text { Visual test } \\
\text { Positive }\end{array}$ & 2 & 3 & 5 & $2 / 5$ & \\
Negative & 14 & 81 & 95 & & \\
Totals & 16 & 84 & 100 & & $(80.0 \%)$ \\
Sensitivity & $2 / 19$ & & & & \\
Specificity & $(12.5 \%)$ & & & & \\
& \multicolumn{5}{c}{$81 / 84$} \\
& $(96.4 \%)$ & & & \\
\hline
\end{tabular}

poor sensitivity the negative predictive value was relatively low. The calculated specificity of $96.5 \%$ is relatively high and was mostly due to the low frequency of positive tests among the samples. A positive result was more likely to be a false positive associated with culture growth of bacteria not associated with UTI.

Limitations of this study include that the urine clarity assessments were performed by a single assessor. Also, samples obtained from a clinical laboratory may differ somewhat from those obtained at the POC in an ambulatory setting.

We concluded that a visual test of urine clarity is not sufficiently accurate to rule out UTI in women.

The authors would like to acknowledge the University of Toledo Medical Center laboratory technicians who read the results of the urine cultures.

\section{References}

1. Sultana RV, Zalstein S, Cameron P, Campbell D. Dipstick urinalysis and the accuracy of the clinical diagnosis of urinary tract infection. J Emerg Med 2001;20(1):13-9.

2. Bulloch B, Bausher JC, Pomerantz WJ, Connors M, Mahabee-Gittens M, Dowd MD. Can urine clarity exclude the diagnosis of urinary tract infection? Pediatrics 2000;106(5):E60. 